\title{
Detection of Vancomycin Resistant Enterococci in Various Clinical Sample Isolates from a Tertiary Care Centre
}

\author{
E. Manimala*, I.M. Rejitha and C. Revathy \\ Department of Microbiology, Tirunelveli Medical college, Tirunelveli-627011, \\ Tamil Nadu, India \\ *Corresponding author
}

\begin{tabular}{|l|}
\hline Ke y w or d s \\
Enterococci, \\
Vancomycin \\
resistant \\
Enterococci,Entero \\
cocci fecalis
\end{tabular}

A B S T R A C T

Enterococci are Gram-positive, facultative anaerobic organisms, ovoid in shape, short chains, in pairs or single cells. The common enterococci-associated nosocomial infections are urinary tract infections, surgical wound infections and bacteremia. Resistance to vancomycin is now widely reported worldwide. In India, the prevalence of VRE has been reported as' $8 \%, 5.5 \%$ and $23 \%$ in New Delhi, Chandigarh, and Mumbai. This study was done to detect the presence of vancomycin resistant enterococci (VRE) among the various samples in a tertiary care hospital. A total of 100 non duplicate isolates of Enterococci were collected from different clinical samples. Antibiotic susceptibility testing was done by Kirby Bauer disc diffusion method. Vancomycin resistance was detected using Vancomycin screen agar and E strip method. Out of 100 Enterococcal isolates, 66 isolates were found to be multi drug resistant 4 isolates (4\%) were presumptively identified as vancomycin resistant by vancomycin screen agar containing $6 \mu \mathrm{g} / \mathrm{ml}$ vancomycin and $\mathrm{E}$ strip method. All the 4 VRE isolates were resistant to Penicillin and Ciprofloxacin. 50\% percent of the isolates were sensitive for High level Gentamycin and Teicoplanin. 100\% percent of the isolates were sensitive to Linezolid and Quinipristin. Enterococci are emerging as an important pathogen causing variety of nosocomial infections and also cause community acquired infections contributing significantly to patients morbidity and mortality. The emergence of Vancomycin resistant Enterococci worsens the problem further because of the Multidrug resistance exhibited by these agents leaving fewer therapeutic options for the clinicians in treating the serious life threatening VRE infections.

\section{Introduction}

The genus Enterococcus consists of Grampositive, facultatively anaerobic organisms that are spectacle shaped and may appear on smear in short chains, in pairs or as single cells. Enterococci, though commensals in adult feces are essential nosocomial pathogens. Enterococcal infections may of at least 12 species including Enterococcus faecalis, E. faecium, E. durans, E. avium, E. casseliflavus, E. gallinarum, E. hirae, E.malodoratus, E. mundtii, E. pseudoavium, E. raffinosus, and E. solitarius. Among enterococcal species, E. faecalis and $E$. faecium are the two major human pathogens 
accounting for $85-89 \%$ and $10-15 \%$ of all enterococcal infections, respectively. Prior to the 1990s also, enterococci have been recognized as an important cause of bacterial endocarditis for almost a century. However, recently they are recognized as a cause of nosocomial infection and "superinfection" in patients receiving antimicrobial agents. The most common Enterococci-associated nosocomial infections are of the urinary tract, followed by surgical site infections and bacteremia.

The intrinsic antibiotic resistance pattern of Enterococci, along with their promiscuity in acquisition and dissemination of genetically versatile antibiotic resistance elements, presents serious challenges to the treatment of enterococcal infections. Infections by Enterococci have traditionally been treated with cell wall active agents (e.g., penicillin or ampicillin) in combination with an aminoglycoside (streptomycin/gentamicin), More ever, emergence of high level aminoglycoside resistance (HLAR), lactam antibiotics and to vancomycin by some strains has led to failure of synergistic effects of combination therapy.

Vancomycin is an effective antimicrobial for treating infection caused by gram positive organisms. Gram positive isolates are often routinely tested for vancomycin susceptibility. In the 1970s, hospital-associated enterococcal infections in the United States were mainly due to E. faecalis. More recently, E. faecium has emerged as therapeutically challenging organism because of its resistance to vancomycin and penicillin. These VRE isolates also have a high level of resistance to aminoglycosides Resistance to glycopeptides is mediated by alteration of the drug target from D-alanine-D-alanine to D-alanine-Dlactate.

The CLSI recommends screening of enterococci for high level aminoglycoside resistance with both gentamicin and streptomycin isolated from blood cultures or specimens such as heart valve tissue. The emergence of multidrug resistant Enterococci, especially Vancomycin Resistant Enterococci (VRE), and its spread has caused the occurrence of many hospitals out breaks worldwide. In the United States, vancomycinresistance Enterococcus faecium accounted for 4 per cent of healthcare-associated infections. It is the second most common pathogen causing mortality and morbidity and the 3rd leading cause of hospital acquired blood stream infection.

The prevalence in Asian countries is decreased and probably due to recent emergence of this resistance in this continent and only a handful of studies to document. In India, the prevalence of VRE has been reported as $8 \%, 5.5 \%$ and $23 \%$ in New Delhi, Chandigarh, and Mumbai, respectively, all of vanB phenotype.

Enterococci have emerged as the leading causes of Multiple drug resistant hospitalacquired pathogens especially with the emergence of glycopeptide-resistant Enterococcus (GRE) species.

\section{Materials and Methods}

This study was conducted during the period from April 2017 to May 2018 at the Department of Microbiology, Tirunelveli Medical College, Tirunelveli. During the study a total of about 100 non duplicate clinical isolates of Enterococci were collected from different clinical samples like clinical specimens such as urine, blood,pus, tissue fluids obtained from both in-patient and outpatient departments of Tirunelveli Medical College. The isolates were identified by standard biochemical tests. Antibiotic susceptibility testing was done by Kirby 
Bauer disc diffusion method. Vancomycin resistance was detected using Vancomycin screen agar and Hicomb E strip method.

\section{Statistical analysis}

Data collected were entered in Microsoft Excel and analysed using statistical analysis software Statistical Package for Social Services (SPSS) v.16. Appropriate descriptive and inferential statistics were calculated. P values $<0.05$ were statistically significant.

\section{Results and Discussion}

Among the 100 enterococcal isolates, 56 isolates were from male patients and 44 from female patients. Most of the isolates (27\%) were from patients aged between 16 and 30 years. The mean age of the patients was found to be 35.6 years with a SD of 21.5 years. The age of the patients ranged from a minimum of 2 years to a maximum age of 84 years.

Out of the 100 samples, 69 samples were collected from inpatients and remaining 31 from outpatients.

Enterococci were commonly isolated from urine $(93 \%)$, followed by sputum (4\%), ascitic fluid (1\%), pus from leg ulcer (1\%) and vaginal swab (1\%) (Table 1).

The isolates were identified to be Enterococcus faecalis (90\%) and Enterococcus faecium (10\%). All the four sputum isolates and pus isolates were found to be E. faecalis whereas the ascitic fluid isolate and vaginal swab isolate was found to be $E$. faecium.

The table 2 shows the antibiotic susceptibility pattern of the enterococcal isolates by Kirby bauer disc diffusion method on Mueller Hinton agar according to CLSI guideline Highest prevalence of resistance was observed against Penicillin (75\%), followed by Ciprofloxacin (59\%) and Vancomycin (48\%). On the other hand, Doxycycline was found to be sensitive for $80 \%$ of isolates, followed by Chloramphenicol (78\%) and High level Gentamicin (66\%). Out of the 100 enterococcal isolates, 66 isolates were found to be multi drug resistant (resistant to three or more antibiotics).

Vancomycin screen agar and E strip method showed only four isolates to be resistant against Vancomycin. Whereas, disc diffusion showed 48 isolates to be resistant against Vancomycin (Table 3). This shows that the disc diffusion method is highly sensitive in detecting resistant isolates of Enterococcus $(100 \%)$. But its specificity in detecting the resistance against Vancomycin remains low $(54 \%)$.

Nearly $10 \%$ of E. faecium was found to be resistant to vancomycin, whereas only $3 \%$ of $E$. faecalis was found to be resistant to vancomycin. There is no statistically significant difference in vancomycin susceptibility between the two species (Table 4).

All the four vancomycin resistant enterococcal isolates were also found to be resistant for penicillin and ciprofloxacin. Fifty percent of the isolates were sensitive for High level Gentamycin and Teicoplanin. All the 4 isolates were sensitive to Linezolid and Quinipristin

Enterococcus species have been recognised as a pathogen causing diseases like bacteremia, endocarditis, complicated urinary tract infections, intra abdominal infections, pelvic infections, wound and soft tissue infections etc. VRE has become an important nosocomial pathogen because of its rapid spread, high mortality rates associated with infections, limited option for treatment, and 
the possibility of transferring vancomycin resistance genes to other more virulent and more prevalent pathogens such as Staphylococcus aureus. This study was conducted to detect prevalence of vancomycin resistant enterococci in clinical isolates by phenotypic and genotypic methods.

A total of 100 enterococcal isolates were collected from 996 culture positive samples received over one year period.

$90 \%$ of the isolates were identified to be $E$. faecalis and $10 \%$ was E. faecium. Both these species are significantly associated with clinical disease, Parameswarappa et al., in their study have found E. faecalis to be the predominant isolate followed by E. faecium. Chakraborthy et al., also reported $E$. faecalisas the predominant Enterococcal species $(80 \%-90 \%)$ in their study followed by E. faecium (5\%-15\%). Several species of enterococci are currently recognized, but generally $90 \%$ of enterococcal infections are caused by E. faecalis and $10 \%$ are caused by E. faecium. Although a few studies have documented an increase in the prevalence of E. faecium, in this study the prevalence of this species was considerable low. E. faecium infections have been found to be more resistant to penicillin and aminoglycosides which is attributed to the production of enzyme 6-acetyl transferase and more penicillin binding proteins.

Resistance to many antimicrobial drugs complicates the treatment of enterococcal infections. Acquired resistance to high concentrations of ampicillin, aminoglycoside, and glycopeptides antibiotics, specifically vancomycin, has exacerbated this problem. Highest prevalence of resistance was observed against penicillin (75\%) followed by ciprofloxacin (59\%) and vancomycin (48\%). Of the 100 isolates, $66 \%$ were multiple drug resistant. Both E. faecalis and E. faecium exhibited $>59 \%$ resistance for ciprofloxacin in this study. High level of ciprofloxacin resistance has been reported by Anbumani et al., and Shah et al., where ciprofloxacin resistance accounted for $58 \%$ and $62 \%$ respectively. The isolates in this study showed $80 \%$ sensitivity to doxycycline.

High level gentamicin resistance (HLGR) was observed in $34 \%$ of the enterococcal isolates in our study. This finding correlates with the Anbumani et al., Shah et al., and Fernandez at al studies where HLGR was 56\%, 53\% and $53 \%$ respectively. Studies conducted in New Delhi and Mumbai have reported HLGR prevalence to be as high as 70 and 100 percent, respectively.

In this study, the occurrence of HLGR among the enterococcal isolates had no significant difference seen between $E$. faecalis and $E$. faecium isolates. However Mendiratta et al., have reported greater resistance to HLG among E. faecium as compared to E. faecalis isolates.

Initial screening for VRE by disc diffusion methods detected 48 isolates as vancomycin resistant. However MIC detection by Hicomb E strip method and Vancomycin screen agar method detected only 4 isolates as vancomycin resistant. These findings suggest that through the disc diffusion method is highly sensitive in detecting resistant isolates of Enterococcus (100\%) its specificity is low $(54 \%)$

In this study the VRE isolation was $4 \%$. Studies from Indore and Nagpur reported 14.29 and $11.38 \%$ VRE, respectively (Chitin et al., Rahangdale et al.,). In India, the prevalence of VRE has been reported to be between 0 and $30 \%$. In our study, among the VRE 3 were E. faecalis 1 was E. faecium. This is similar to the finding by Agarwal et al., who found vancomycin resistance to be 
greater among E. faecalis isolates.

Table.1 Distribution of the Enterococcal isolates in different clinical samples

\begin{tabular}{|c|c|}
\hline Sample & Percentage \\
\hline Urine & 93 \\
\hline Sputum & 4 \\
\hline Pus & 1 \\
\hline Vaginal swab & 1 \\
\hline Ascitic fluid & 1 \\
\hline
\end{tabular}

Table.2 Antimicrobial sensitivity pattern of the Enterococcal isolates by disc diffusion method $(\mathrm{N}=100)$

\begin{tabular}{|l|c|c|}
\hline Antibiotic & Sensitive isolates (\%) & Resistant isolates (\%) \\
\hline Penicillin & $25(25 \%)$ & $75(75 \%)$ \\
\hline Tetracycline & $60(60 \%)$ & $40(40 \%)$ \\
\hline Ciprofloxacin & $41(41 \%)$ & $59(59 \%)$ \\
\hline Chloramphenicol & $78(78 \%)$ & $22(22 \%)$ \\
\hline Doxycycline & $80(80 \%)$ & $20(20 \%)$ \\
\hline High level Gentamycin & $66(66 \%)$ & $34(34 \%)$ \\
\hline Vancomycin & $52(52 \%)$ & $48(48 \%)$ \\
\hline
\end{tabular}

Table.3 Vancomycin susceptibility pattern by different methods

\begin{tabular}{|c|c|}
\hline Testing method & \% of VRE \\
\hline Disc diffusion method & 48 \\
\hline Vancomycin screen agar & 4 \\
\hline E strip method & 4 \\
\hline
\end{tabular}

Table.4 Antimicrobial susceptibility of the Vancomycin resistant isolates

\begin{tabular}{|c|c|c|}
\hline Antibiotic & Sensitive & Resistant \\
\hline Linezolid & 4 & - \\
\hline Teicoplanin & 2 & 2 \\
\hline Quinipristin & 4 & - \\
\hline High level Gentamycin & 2 & 2 \\
\hline Penicillin & - & 4 \\
\hline Ciprofloxacin & - & 4 \\
\hline Doxycycline & 1 & 3 \\
\hline
\end{tabular}

Of the $4 \mathrm{VRE}$ isolates $2 \mathrm{VRE}$ were found to be susceptible to high level gentamicin. Hence, that infection could be treated with a combination of a high level aminoglycoside and a lactam antibiotic. The presence of high level gentamycin resistance and concurrent resistance to Penicillin or Ampicillin and vancomycin has been reported in some 
studies. An USA based study demonstrated that gentamycin resistance plasmid might cotransfer vancomycin resistance plasmids. Hence the detection of high level gentamycin resistance along with vancomycin resistant enterococci represents a significant problem in this region.

On studying the susceptibility pattern of VRE isolates to supplemental drugs like linezolid, Quinupristin and chloramphenicol, all of the he VRE isolates were susceptible to Linezolid and Quinipristin ( $100 \%$ sensitivity). $100 \%$ of the isolates showed resistance to chloramphenicol. The study carried out by Gupta et al., from Chandigarh, India and MM Salem Behkit et al., from Iran have reported, $100 \%$ sensitivity of VRE isolates to linezolid which is similar to our study. Agarwal et al., has also reported $100 \%$ sensitivity to linezolid in their study. Perlada et al., from Australia also have reported $100 \%$ sensitivity to linezolid and $100 \%$ sensitivity to Chloramphenicol. But in our study $100 \%$ of VRE isolates were resistant to Chloramphenicol.

In conclusion, Enterococci are emerging as an important pathogen causing variety of nosocomial infections and also cause community acquired infections contributing significantly to patient's morbidity and mortality.

The emergence of Vancomycin resistant Enterococci worsens the problem further because of the Multidrug resistance exhibited by these agents leaving fewer therapeutic options for the clinicians in treating the serious life threatening VRE infections.

The prevalence of VRE varies based on geographic location, antibiotic use of the subject population. This study emphasises the need for conducting frequent surveillance. Programmes for prompt identification of VRE in hospitals and community.

This also highlights the need for implementation of stringent infection control measures like rational use of antibiotics especially restricting the use of Vancomycin to minimum, proper containment and effective treatment of VRE infections, strict hand washing practices, education of the healthcare workers and other personnel involved in the patient management. These measures are to be strictly followed to bring down the mortality and morbidity associated with these hospital acquired VRE infections.

\section{Acknowledgement}

The authors are gratefully acknowledge. The Dean, Tirunelveli Medical college Hospital, Tirunelveli, Tamil Nadu and The staff of Microbiology, Microbiology Department of Tirunelveli Medical college Hospital.

\section{References}

1. Anbumani Narayanaswamy et al., Speciation and antimicrobial susceptibility pattern of Enterococci from a tertiary health care centre of south India. Journal of pharmacy Research, 2011; 2: 25-7.

2.Agarwal J, Kalyan R, Singh M. High-level aminoglycoside resistance and $\beta$ lactamase production in Enterococci at a tertiary care hospital in India. Jpn $J$ Infect Dis 2009; 62:158-9.

3. Chitin S, Katara G, Hemvani N, Pareek S, Chitnis DS. In vitro activity of daptomycin \& linezolid against methicillin resistant Staphylococcus aureus \& vancomycin-resistant enterococci isolated from hospitalized cases in Central India. Indian J Med Res 2013; 137: 191-6.

4.Gupta V, Singla N. Antibiotic susceptibility pattern of enterococci. J ClinDiagn Res 
2007; 5: 385-9.

5.Mendiratta D, Kaur H, Deotale V, Thamke DC, Narang R, Narang P. Status of high level aminoglycoside resistant Enterococcus faecium and Enterococcus faecalis in a rural hospital of central India. Indian J Med Microbiol 2008; 26: 369-71.

6. Murray BE. The life and times of the Enterococcus. Clin Microbial Rev 1990; 3:46-65. Back to cited text no.1

7. Parvathi S, Appalaraju B. Isolation, characterisation and antibiogram of Enterococci from clinical samples. Indian J pathol Microbial 2003; 46: 501-3 Back to cited text no.2

8. Parameswarappa J, Basavaraj VP, Basavaraj CM. Isolation, identification and antibiogram of enterococci isolated from patients with urinary tract infection. Annaafr med. 2013; 12: 17681.
9. Sood S, Malhotra M, Das BK, Kapil A. Enterococcal infections \& antimicrobial resistance. Indian Med Res 2008; 128: 111-21.

10. Salem Bekhit MM, Moussa I, Muharram MM, Alanazy FK, HM. Prevalence and antimicrobial resistance pattern of multidrug- resistant enterococci isolates from clinical specimens. Indian J Med Microbiol., 2012; 30: 44-51.

11. Ross PW. Streptococcus and Enterococcus. In colle JG, Fraser BP, Marimion BP, et al., editors. Mackie and McCartney practical Medical Microbiology. $14^{\text {th }}$ ed. London: Churchill Livingstone; 1996. p. 263-74. Back to cited text no 4.

12. Rahangdale VA, Agrawal G, Jalgaonkar SV. Study of antimicrobial resistance in enterococci. Indian J Med. Microbiol., 2008; 26: 285-7.

\section{How to cite this article:}

Manimala, E., I.M. Rejitha and Revathy, C. 2019. Detection of Vancomycin Resistant Enterococci in Various Clinical Sample Isolates from a Tertiary Care Centre. Int.J.Curr.Microbiol.App.Sci. 8(02): 915-921. doi: https://doi.org/10.20546/ijcmas.2019.802.104 